\title{
Pacific
}

Journal of

Mathematics

\section{INDEFINITE KAC-MOODY ALGEBRAS OF SPECIAL LINEAR} TYPE

Georgia Benkart, Seok-Jin Kang and Kailash C. Misra 


\title{
INDEFINITE KAC-MOODY ALGEBRAS OF SPECIAL LINEAR TYPE
}

\author{
Georgia Benkart, Seok-Jin Kang, Kailash C. Misra
}

From the special linear Lie algebra $A_{n}=s \ell(n+1, \mathbb{C})$ we construct certain indefinite Kac-Moody Lie algebras $I A_{n}(a, b)$ and then use the representation theory of $A_{n}$ to determine explicit closed form root multiplicity formulas for the roots $\alpha$ of $I A_{n}(a, b)$ whose degree satisfies $|\operatorname{deg}(\alpha)| \leq 2 a+1$. These expressions involve the well-known Littlewood-Richardson coefficients and Kostka numbers. Using the Euler-Poincaré Principle and Kostant's formula, we derive two expressions, one of which is recursive and the other closed form, for the multiplicity of an arbitrary root $\alpha$ of $I A_{n}(a, b)$ as a polynomial in Kostka numbers.

\section{Introduction.}

For Kac-Moody algebras the root multiplicities of only the finite and affine algebras are explicitly known. In this paper, the third of a series of articles on the structure of non-finite, non-affine Kac-Moody algebras, we study certain indefinite Kac-Moody algebras coming from the special linear Lie algebra $A_{n}=s \ell(n+1, \mathbb{C})$ of traceless $(n+1) \times(n+1)$ complex matrices. The main theme of these articles is that combinatorial results from the representation theory of classical simple Lie algebras can be applied to the problem of determining root multiplicities for Kac-Moody algebras. The starting point is a well-known construction of graded Lie algebras of Kac-Moody type whose ingredients are a Lie algebra $G$ over $\mathbb{C}$, two $G$-modules $V$ and $V^{\prime}$, and a $G$ module homomorphism $\psi: V^{\prime} \otimes V \rightarrow G$. The graded Lie algebra $\mathcal{L}=$ $\mathcal{L}\left(G, V, V^{\prime}, \psi\right)=\sum_{k \in \mathbb{Z}} \mathcal{L}_{k}$ built from these components contains no graded ideals which intersect the local part $V \oplus G \oplus V^{\prime}$ trivially. The algebra $G$ is specialized to be $g \ell(n+1, \mathbb{C})=s \ell(n+1, \mathbb{C}) \oplus \mathbb{C} I$. The $G$-module $V$ is assumed to be $V\left(b \Lambda_{1}\right)=V\left(b \epsilon_{1}\right)$, the irreducible $G$-module with highest weight $b$ times the first fundamental weight $\Lambda_{1}$, or equivalently $b$ times $\epsilon_{1}$, where $\epsilon_{1}$ maps a matrix to its $(1,1)$ entry. The homomorphism $\psi$ is the map given by (2.1) below. A certain parameter " $a$ " enters into the definition of $\psi$. We argue that the algebra $\mathcal{L}\left(G, V, V^{*}, \psi\right)$ is isomorphic to the Kac-Moody algebra having generalized Cartan matrix 


$$
\mathcal{C}=\left(\begin{array}{ccccc}
2-b & 0 & \cdots & 0 \\
-a & & & \\
0 & & C\left(A_{n}\right) \\
\vdots & & & \\
0 & & &
\end{array}\right)
$$

where $C\left(A_{n}\right)$ is the Cartan matrix of Lie algebra $A_{n}$. For almost all positive integral values of $a$ and $b$, the matrix $\mathcal{C}$ is of indefinite type, which we denote by $I A_{n}(a, b)$.

Our investigations in [BKM2] focused on the $b=1$ case where we determined closed form formulas for the multiplicities of roots $\alpha=k \alpha_{0}+k_{1} \alpha_{1}+$ $\cdots+k_{n} \alpha_{n}$ whose degree $k$ satisfies $-2 a \leq k \leq 2 a$. We also considered in that paper the analogous indefinite algebras $I B_{n}(a, 1), I C_{n}(a, 1)$, and $I D_{n}(a, 1)$ constructed from an algebra $G$ which is a central extension of a simple Lie algebra of type $B_{n}, C_{n}$ or $D_{n}$ respectively.

Section 1 of this present work reviews the basic construction and background results. In the next section the construction is specialized and is shown to give the indefinite algebras $I A_{n}(a, b)$. In the third section we develop closed form multiplicity formulas for the roots of $I A_{n}(a, b)$ up to degree $2 a+1$, that is, for all roots in the graded components $\mathcal{L}_{k}$ for $k=0, \pm 1, \ldots, \pm(2 a+1)$. The multiplicity formulas involve the well-known Littlewood-Richardson coefficients and Kostka numbers and are similar in spirit to the ones found in [BKM2] for the case $b=1$ and in [BKM1] for the case $n=1$. However, there are added complications which must be dealt with here in going from the $b=1$ case to the general case.

In the final section we use the Euler-Poincare Principle and Kostant's formula to derive two expressions, one of which is recursive and the other closed form, for the root multiplicities of the Kac-Moody algebras $I A_{n}(a, b)$. The closed form formula we obtain is related to the Berman-Moody formula $[\mathbf{B M}]$ in that ours corresponds to a maximal proper subset of the simple roots, while the Berman-Moody formula corresponds to the empty subset. This connection is explained further in [Kan3]. These formulas enable us to write the multiplicity of an arbitrary root of $I A_{n}(a, b)$ as a polynomial in Kostka numbers.

Many interesting Kac-Moody algebras are indefinite Kac-Moody algebras of special linear type. For example, the rank two Kac-Moody algebras are just the algebras $I A_{1}(a, b)$. The hyperbolic algebra $H A_{1}^{(1)}$ studied by Feingold and Frenkel [FF] and Kang [Kan1] [Kan2] is $I A_{2}(2,2)$ in our notation, and the hyperbolic algebra $H A_{2}^{(2)}$, whose root multiplicities have been investigated in [Kan2], is $I A_{2}(4,1)$. Similarly, the hyperbolic algebra $H G_{2}^{(1)}$ 
is $I A_{3}(3,1)$ and $H D_{4}^{(3)}$ is $I A_{3}(1,3)$.

\section{The Construction.}

We begin this section by recalling some necessary background results which can be found in [BKM1]. The first is the construction whose basic ingredients are a Lie algebra $G$, two $G$-modules $V$ and $V^{\prime}$, and a $G$-module homomorphism $\psi: V^{\prime} \otimes V \longrightarrow G$. Set $\mathcal{F}_{0}=G, \mathcal{F}_{-1}=V$, and $\mathcal{F}_{1}=V^{\prime}$. Let $\mathcal{F}^{-}=\sum_{k \geq 1} \mathcal{F}_{-k}$ (resp. $\mathcal{F}^{+}=\sum_{k \geq 1} \mathcal{F}_{k}$ ) be the free Lie algebra generated by $\mathcal{F}_{-1}$ (resp. $\mathcal{F}_{1}$ ). Then $\mathcal{F}_{-k}$ (resp. $\mathcal{F}_{k}$ ) for $k>1$ is the space of all products of $k$ vectors from $\mathcal{F}_{-1}$ (resp. $\mathcal{F}_{1}$ ). In particular, the set of elements $\left[x_{1}\left[x_{2}, \cdots,\left[x_{k-1}, x_{k}\right] \ldots\right]\right]$, where the vectors $x_{i}$ are chosen from a basis for $\mathcal{F}_{-1}$ (resp. $\left.\mathcal{F}_{1}\right)$, spans $\mathcal{F}_{-k}$ (resp. $\mathcal{F}_{k}$ ). There is a Lie bracket for $\mathcal{F}=\mathcal{F}^{-} \oplus \mathcal{F}_{0} \oplus \mathcal{F}^{+}$which extends the products in $\mathcal{F}^{-}, \mathcal{F}^{+}$, and $\mathcal{F}_{0}$. Thus, for $g \in \mathcal{F}_{0}, v \in \mathcal{F}_{-1}$, and $w \in \mathcal{F}_{1}$,

$$
[g, x]=g \cdot x=-[x, g] \quad \text { if } x=v, w
$$

and

$$
[w, v]=\psi(w \otimes v)=-[v, w] .
$$

Under this bracket, $\mathcal{F}=\sum_{k \in \mathbb{Z}} \mathcal{F}_{k}$ becomes a graded Lie algebra which is generated by its local part $\mathcal{F}_{-1}+\mathcal{F}_{0}+\mathcal{F}_{1}$.

For $k>1$ define the subspaces

$$
J_{ \pm k}=\left\{x \in \mathcal{F}_{ \pm k} \mid\left[y_{1},\left[\cdots\left[y_{k-1}, x\right]\right] \cdots\right]=0 \quad \text { for all } y_{1}, \ldots, y_{k-1} \in \mathcal{F}_{\mp 1}\right\}
$$

and set

$$
J^{-}=\sum_{k>1} J_{-k}, \quad J^{+}=\sum_{k>1} J_{k} .
$$

Then by ([BKM1], Proposition 1.7 or [FF], Proposition 4.2) $J^{-}$and $J^{+}$ are ideals of $\mathcal{F}$, and the ideal $J=J^{-} \oplus J^{+}$is the largest graded ideal of $\mathcal{F}$ trivially intersecting $\mathcal{F}_{-1}+\mathcal{F}_{0}+\mathcal{F}_{1}$. Our main object of study is the graded Lie algebra

$$
\begin{aligned}
\mathcal{L} & =\mathcal{L}\left(G, V, V^{\prime}, \psi\right) \stackrel{\text { def }}{=} \mathcal{F}^{-} / J^{-} \oplus \mathcal{F}_{0} \oplus \mathcal{F}^{+} / J^{+} \\
& =\cdots \oplus \mathcal{L}_{-2} \oplus \mathcal{L}_{-1} \oplus \mathcal{L}_{0} \oplus \mathcal{L}_{1} \oplus \mathcal{L}_{2} \oplus \cdots
\end{aligned}
$$

where $\mathcal{L}_{i}=\mathcal{F}_{i}$ for $i= \pm 1,0$ and $\mathcal{L}_{i}=\mathcal{F}_{i} / J_{i}$, for $i \neq \pm 1,0$. The algebra 
$\mathcal{L}=\oplus \sum_{k \in \mathbb{Z}} \mathcal{L}_{k}$ has no graded ideals which trivially intersect the local part $V \oplus G \oplus V^{\prime}$.

Let $\mathcal{L}^{ \pm}=\mathcal{F}^{ \pm} / J^{ \pm}=\sum_{k \geq 1} \mathcal{L}_{ \pm k}$, and for a fixed choice of $G, V, V^{\prime}, \psi$, let $m \geq 2$ denote the smallest integer such that $J_{-m} \neq(0)$. Then $J_{m} \neq(0)$ must hold. We will consider cases where $J^{-}=\sum_{k \geq m} J_{-k}$ (resp. $J^{+}=\sum_{k \geq m} J_{k}$ ) is the graded ideal of $\mathcal{F}^{-}$(resp. $\left.\mathcal{F}^{+}\right)$generated by $J_{-m}$ (resp. $\left.J_{m}\right)$.

Suppose now that $G=g \ell(n+1, \mathbb{C})=s \ell(n+1, \mathbb{C}) \oplus \mathbb{C} I$ and let $\mathcal{H}$ be the Cartan subalgebra of diagonal matrices in $G$. Assume $V$ is a faithful irreducible highest weight $G$-module relative to $\mathcal{H}$. Then the dual space $V^{*}$ of $V$ is a lowest weight module for $G$. The free Lie algebra $\mathcal{F}^{+}$(resp. $\mathcal{F}^{-}$) generated by $\mathcal{F}_{1}=V^{*}$ (resp. $\mathcal{F}_{-1}=V$ ) is a module for $\mathcal{F}_{0}=G$ with finite dimensional weight spaces relative to $\mathcal{H}$, and the multiplicities of those weight spaces can be computed using the following generalization of the Witt formula.

Proposition 1.6 ([Kan1], [Kan2]). Let $\Omega=\left\{\omega_{1}, \omega_{2}, \ldots\right\}$ be an enumeration of the weights of $\mathcal{F}_{-1}=V$ relative to $\mathcal{H}$. Then for any weight $\gamma$ of $\mathcal{F}^{-}$,

$$
\operatorname{dim} \mathcal{F}_{\gamma}^{-}=\sum_{\omega \mid \gamma} \mu\left(\frac{\gamma}{\omega}\right) \frac{\omega}{\gamma} D(\omega)
$$

where $\mu$ denotes the classical Möbius function; $\omega \mid \gamma$ if $\gamma=\kappa \omega$ for some positive integer $\kappa$, in which case $\frac{\gamma}{\omega}=\kappa$ and $\frac{\omega}{\gamma}=\frac{1}{\kappa}$; and

$$
D(\omega)=\sum_{(t) \in T(\omega)} \frac{\left(\left(\sum_{i} t_{i}\right)-1\right) !}{\prod_{i}\left(t_{i} !\right)} \prod_{i}\left(\operatorname{dim} V_{\omega_{i}}\right)^{t_{i}}
$$

where $T(\omega)=\left\{(t)=\left(t_{1}, t_{2}, \ldots\right) \mid t_{i} \in \mathbb{Z}^{\geq 0}\right.$ and $\left.\sum_{i} t_{i} \omega_{i}=\omega\right\}$.

The algebra $\mathcal{L}=\mathcal{L}\left(G, V, V^{*}, \psi\right)$ has finite dimensional root spaces relative to $\mathcal{H}$, and to compute the multiplicities of those roots we need additional information about the spaces $J_{ \pm k}$ in the ideal $J$. This information comes from considering the homology module $H_{3}\left(\mathcal{L}^{-}\right)$which inherits a $\mathbb{Z}$-grading from that of $\mathcal{L}^{-}=\mathcal{F}^{-} / J^{-}$. Suppose that $d$ is the smallest integer with $H_{3}\left(\mathcal{L}^{-}\right)_{-d} \neq(0)$. As Kang shows in ([Kan1], [Kan2]), the value of $d$ determines the structure of certain of the homogeneous components of $J$ :

Proposition 1.9. Let $\mathcal{F}^{-}=\sum_{k \geq 1} \mathcal{F}_{-k}$ be the free Lie algebra generated by the $G$-module $\mathcal{F}_{-1}=V$. Let $J^{-}=\sum_{k \geq m} J_{-k}$ be the ideal of $\mathcal{F}^{-}$generated by $J_{-m} \subseteq \mathcal{F}_{-m}$ for some $m \geq 2$. For $\hat{\mathcal{L}}^{-}=\mathcal{F}^{-} / J^{-}$let $d$ be the smallest integer such that $H_{3}\left(\mathcal{L}^{-}\right)_{-d} \neq(0)$. Then

(1) for $m \leq j<\min (2 m, d)$, 


$$
J_{-j} \cong \underbrace{V \otimes \cdots \otimes V}_{(j-m) \text { times }} \otimes J_{-m}
$$

(2) If $d<2 m$, then

$$
J_{-d} \cong(\underbrace{V \otimes \cdots \otimes V}_{(d-m) \text { times }} \otimes J_{-m}) / H_{3}\left(\mathcal{L}^{-}\right)_{-d} .
$$

In the next section we consider the algebra $\mathcal{L}\left(G, V, V^{*}, \psi\right)$ under more stringent restrictions on the module $V$.

\section{Indefinite Kac-Moody Algebras of Type $\operatorname{IA}_{n}(\mathbf{a}, \mathbf{b})$.}

The module $V=V\left(b \Lambda_{1}\right)=V\left(b \epsilon_{1}\right)$ for $G=g \ell(n+1, \mathbb{C})$ can be explicitly realized as the $\mathbb{C}$-vector space of homogeneous polynomials of total degree $b$ in the indeterminates $z_{1}, \ldots, z_{n+1}$. We adopt the shorthand $z^{\underline{r}}$ for the monomial $z_{1}^{r_{1}} z_{2}^{r_{2}} \cdots z_{n+1}^{r_{n+1}}$ corresponding to the $(n+1)$-tuple $\underline{r}=\left(r_{1}, r_{2}, \ldots, r_{n+1}\right)$, and let $\xi_{i}$ be the $(n+1)$-tuple with 1 in the $i$ th position and 0 elsewhere. Then the action of the matrix unit $E_{i, j}$ in $g \ell(n+1, \mathbb{C})$ on $z^{\underline{r}}$ is afforded by

$$
E_{i, j} z^{\underline{r}}=r_{j} z^{\underline{\underline{r}+\xi_{i}-\xi_{j}}}
$$

where it is understood that $z^{\underline{r}+\xi_{i}-\xi_{j}}$ is 0 if any component of the $(n+1)$-tuple $\underline{r}+\xi_{i}-\xi_{j}=\left(r_{1}, \ldots, r_{i}+1, \ldots, r_{j}-1, \ldots, r_{n+1}\right)$ is negative. Thus, $E_{i, j}$ acts as $z_{i} \partial / \partial_{z_{j}}$. Assume $V^{*}$ is the dual space of $V$, and let $\left\{\partial^{\underline{s}} \mid \underline{s}=\left(s_{1}, \ldots, s_{n+1}\right)\right.$ and $\left.\sum_{i=1}^{n+1} s_{i}=b\right\}$ be the dual basis to the basis $\left\{z^{\underline{r}}\right\}$ so that $\partial^{\underline{s}}\left(z^{\underline{r}}\right)=\delta_{\underline{s}, \underline{r}}$. We define

$$
\psi\left(\partial^{\underline{s}} \otimes z^{\underline{r}}\right)=\frac{-a}{b} \sum_{i, j=1}^{n+1} r_{i} \delta_{\underline{s}, \underline{r}+\xi_{j}-\xi_{i}} E_{i, j}+\left(a-\frac{2}{b}\right) \delta_{\underline{s}, \underline{r}} I
$$

where $I$ is the identity matrix in $G=g \ell(n+1, \mathbb{C})$. Then $\psi$ is a $G$-module homomorphism, which can be seen by direct computation or by using ([FF], Proposition 4.1) coupled with the fact that the basis of matrix units $E_{i, j}$ forms an orthonormal basis for $G$ relative to the trace form $\left(g, g^{\prime}\right)=\operatorname{tr}\left(g g^{\prime}\right)$.

Suppose $\alpha_{0}=-b \epsilon_{1}$ and $\alpha_{i}=\epsilon_{i}-\epsilon_{i+1}$ for $i=1, \ldots, n$, where $\epsilon_{i}: \mathcal{H} \longrightarrow \mathbb{C}$ is the projection of a matrix in the Cartan subalgebra $\mathcal{H}$ onto its $(i, i)$ entry. Then the monomial $z^{b \xi_{1}}=z_{1}^{b}$ is a maximal vector for $V$ of highest weight 
$b \Lambda_{1}=b \epsilon_{1}$ and $\partial^{b \xi_{1}}$ is a minimal vector of lowest weight $-b \Lambda_{1}=-b \epsilon_{1}$, and the weights of $V$ are given by

$$
\begin{gathered}
\left\{b \Lambda_{1}-k_{1} \alpha_{1}-\cdots-k_{n} \alpha_{n} \mid b \geq k_{1} \geq k_{2} \geq \cdots \geq k_{n} \geq 0\right\}= \\
\left\{m_{1} \epsilon_{1}+m_{2} \epsilon_{2}+\cdots+m_{n+1} \epsilon_{n+1} \mid m_{i} \geq 0 \text { and } m_{1}+\cdots+m_{n+1}=b\right\}
\end{gathered}
$$

Let $e_{i}=E_{i, i+1}, f_{i}=E_{i+1, i}, h_{i}=E_{i, i}-E_{i+1, i+1}$, where $i=1, \ldots, n$, denote the canonical generators for $[G, G]=s \ell(n+1, \mathbb{C})$, and let

Set

$$
e_{0}=\partial^{b \xi_{1}} \quad \text { and } \quad f_{0}=z^{b \xi_{1}}=z_{1}^{b}
$$

$$
h_{0}=\left[e_{0}, f_{0}\right]=-a E_{1,1}+\left(a-\frac{2}{b}\right) I .
$$

Then it is easy to verify using the maximality of $f_{0}=z^{b \xi_{1}}$ and the minimality of $e_{0}=\partial^{b \xi_{1}}$ that the relations

$$
\begin{aligned}
& {\left[h_{i}, h_{j}\right]=0} \\
& {\left[h_{i}, e_{j}\right]=\alpha_{j}\left(h_{i}\right) e_{j}} \\
& {\left[h_{i}, f_{j}\right]=-\alpha_{j}\left(h_{i}\right) f_{j}} \\
& {\left[e_{i}, f_{j}\right]=\delta_{i j} h_{i}}
\end{aligned}
$$

hold in $\mathcal{L}\left(G, V, V^{*}, \psi\right)$. If $\mathcal{C}=\left(\mathcal{C}_{i, j}\right)_{i, j=0}^{n}$ is the matrix whose $(i, j)$ entry is given by $\mathcal{C}_{i, j}=\alpha_{j}\left(h_{i}\right)$, then as in [BKM1] we have,

Theorem 2.4. The Lie algebra $\mathcal{L}\left(G, V, V^{*}, \psi\right)$ with $G=g \ell(n+1, \mathbb{C})$, $V=V\left(b \Lambda_{1}\right)$ and $\psi$ as in (2.1) is isomorphic to the Kac-Moody algebra $I A_{n}(a, b)$ with Cartan matrix

$$
\mathcal{C}=\left(\begin{array}{cccc}
2-b & \cdots & 0 & 0 \\
-a & & & \\
0 & C\left(A_{n}\right) & \\
\vdots & & &
\end{array}\right)
$$

where $C\left(A_{n}\right)$ is the Cartan matrix of $A_{n}=s \ell(n+1, \mathbb{C})$. 


\section{Root Multiplicities in $\mathbf{I A}_{n}(\mathbf{a}, \mathbf{b})$.}

An integral combination $\theta=\theta_{1} \epsilon_{1}+\cdots+\theta_{n+1} \epsilon_{n+1}$ of the $\epsilon_{i}$ 's is a dominant weight of $G=g \ell(n+1, \mathbb{C})$ relative to $\mathcal{H}$ if and only if $\left\{\theta_{1} \geq \theta_{2} \geq \cdots \geq\right.$ $\left.\theta_{n+1} \geq 0\right\}$ determines a partition of $|\theta| \stackrel{\text { def }}{=} \sum_{i=1}^{n+1} \theta_{i}$. Thus, if

$$
\begin{aligned}
\alpha & =-\left(j \alpha_{0}+k_{1} \alpha_{1}+\cdots+k_{n} \alpha_{n}\right) \\
& =\left(j b-k_{1}\right) \epsilon_{1}+\left(k_{1}-k_{2}\right) \epsilon_{2}+\cdots+\left(k_{n-1}-k_{n}\right) \epsilon_{n}+k_{n} \epsilon_{n+1}
\end{aligned}
$$

is a root of $\mathcal{L}=\mathcal{L}\left(G, V, V^{*}, \psi\right) \cong I A_{n}(a, b)$ of degree $-j$, then $\alpha$ is a dominant weight if and only if $\left\{j b-k_{1} \geq k_{1}-k_{2} \geq \cdots \geq k_{n-1}-k_{n} \geq k_{n} \geq 0\right\}$ forms a partition of $j b$ into at most $n+1$ nonzero parts. We identify $\alpha$ with the partition $\left\{\theta_{1} \geq \theta_{2} \geq \cdots \geq \theta_{n} \geq \theta_{n+1} \geq 0\right\}$ having $\theta_{1}=j b-k_{1}$, and $\theta_{i}=k_{i-1}-k_{i}$ for $i \geq 2$ and write $\alpha \vdash j b$ to signify that $\alpha$ determines a partition of $j b$. It suffices to compute the multiplicities of roots that are dominant weights, for the others are conjugate to those under action of the Weyl group of $\mathcal{L}$. It also suffices to determine the multiplicity mult $(\alpha)$ of $\alpha$ for $\alpha$ a root of $\mathcal{L}^{-}$as mult $(-\alpha)=\operatorname{mult}(\alpha)$. Now by Section 1 ,

$$
\begin{aligned}
\operatorname{mult}(\alpha) & =\operatorname{dim} \mathcal{L}_{\alpha} \\
& =\operatorname{dim} \mathcal{L}_{\alpha}^{-} \\
& =\operatorname{dim} \mathcal{F}_{\alpha}^{-}-\operatorname{dim} J_{\alpha}^{-} .
\end{aligned}
$$

Thus, our strategy for computing $\operatorname{mult}(\alpha)$ is to invoke (1.7) for $\operatorname{dim} \mathcal{F}_{\alpha}^{-}$ and to use Proposition 1.9 for $\operatorname{dim} J_{\alpha}^{-}$. The latter involves determining the homology $H_{3}\left(\mathcal{L}^{-}\right)$. Throughout this calculation we use $V(\lambda)$ to denote the irreducible $G$-module with highest weight $\lambda$. In particular, $\mathcal{L}_{-1}=V=$ $V\left(-\alpha_{0}\right)=V\left(b \epsilon_{1}\right)$. Our first result in this direction is

Proposition 3.2. For $1 \leq j \leq a, \mathcal{L}_{-j}=\mathcal{F}_{-j}$, so $\operatorname{mult}(\alpha)=\operatorname{dim}\left(\mathcal{F}_{-j}\right)_{\alpha}$ for all roots of degree $-j$.

Proof. By the Gabber-Kac Theorem [GK], the ideal $J^{-}$of $\mathcal{F}^{-}$is generated by the element $\left(a d f_{0}\right)^{1+a} f_{1}$, which has degree $-(a+1)$ and weight $-(a+$ 1) $\alpha_{0}-\alpha_{1}=(a b+b-1) \epsilon_{1}+\epsilon_{2}$. Hence, $J^{-}$is generated by the space

$$
J_{-(a+1)} \cong V\left(-(a+1) \alpha_{0}-\alpha_{1}\right)=V\left((a b+b-1) \epsilon_{1}+\epsilon_{2}\right) .
$$

The assertions then follow.

Let $\Delta \subset \mathcal{H}^{*}$ be the set of roots of $\mathcal{L}$ and let $\alpha_{0}=-b \epsilon_{1}, \alpha_{i}=\epsilon_{i}-\epsilon_{i+1}$ for $i=1, \ldots, n$, be the simple roots in $\Delta$. Use $\Delta^{+}$(resp. $\Delta^{-}$) to denote 
the positive (resp. negative) roots of $\mathcal{L}$ relative to $\alpha_{0}, \alpha_{1}, \ldots, \alpha_{n}$. The Weyl group $W$ of $\mathcal{L}$ is generated by the set $\left\{s_{i} \mid i=0,1, \ldots, n\right\}$, where $s_{i}$ is the reflection $s_{i}(\gamma)=\gamma-\gamma\left(h_{i}\right) \alpha_{i}$ determined by the simple root $\alpha_{i}$. For $w \in W$, $l(w)$ is the length of $w$ relative to these generators. Let $\Delta_{0}^{+}$(resp. $\Delta_{0}^{-}$) denote the set of positive (resp. negative) roots in $\mathcal{L}$ of degree 0 , so that every root in $\Delta_{0}^{ \pm}$is a combination of $\left\{\alpha_{i} \mid i=1, \ldots, n\right\}$. Set $\Delta_{\neq 0}^{ \pm} \stackrel{\text { def }}{=} \Delta^{ \pm} \backslash \Delta_{0}^{ \pm}$, and let

$$
W^{\prime}=\left\{w \in W \mid w^{-1} \Delta_{0}^{+} \subseteq \Delta^{+}\right\},
$$

as in ([GL], Proposition 8.1). This leads to the following useful lemma:

Lemma 3.4([Kan1], Lemma 4.3). Suppose $w=w^{\prime} s_{j}$ and $l(w)=l\left(w^{\prime}\right)+1$. Then $w \in W^{\prime}$ if and only if $w^{\prime} \in W^{\prime}$ and $w^{\prime}\left(\alpha_{j}\right) \in \Delta_{\neq 0}^{+}$.

By Kostant's formula (see Garland and Lepowsky ([GL], Theorem 8.6) or $\operatorname{Liu}[\mathbf{L i}])$, we have for the homology module $H_{k}\left(\mathcal{L}^{-}\right)$,

$$
H_{k}\left(\mathcal{L}^{-}\right)=\bigoplus \sum_{\substack{w \in W^{\prime} \\ l(w)=k}} V(w \rho-\rho)
$$

where $\rho \in \mathcal{H}^{*}$ satisfies $\rho\left(h_{i}\right)=1$ for $i=0,1, \ldots, n$. Combining these results gives

Proposition 3.6. Suppose that $a \geq 2$ and $b \geq 1$. Then

$$
H_{3}\left(\mathcal{L}^{-}\right)= \begin{cases}V\left(-a(b+1) \alpha_{0}-(b+1) \alpha_{1}\right) & \text { for } \quad n=1 \\ V\left(-a(b+1) \alpha_{0}-(b+1) \alpha_{1}\right) \oplus V\left(-(2 a+1) \alpha_{0}-2 \alpha_{1}-\alpha_{2}\right) & \text { for } n \geq 2\end{cases}
$$

Proof. By (3.5), $H_{3}\left(\mathcal{L}^{-}\right)=\oplus \sum_{\substack{w \in W^{\prime} \\ l(w)=3}} V(w \rho-\rho)$. Using Lemma 3.4 it easy to verify for $n=1$, that $s_{0} s_{1} s_{0}$ is the only element of length 3 in $W^{\prime}$ and for $n \geq 2$, the only elements of length 3 in $W^{\prime}$ are

$$
s_{0} s_{1} s_{0} \quad \text { and } \quad s_{0} s_{1} s_{2} .
$$

Hence, the result follows since

$$
s_{0} s_{1} s_{0} \rho-\rho=-a(b+1) \alpha_{0}-(b+1) \alpha_{1}
$$

and

$$
s_{0} s_{1} s_{2} \rho-\rho=-(2 a+1) \alpha_{0}-2 \alpha_{1}-\alpha_{2} \text {. }
$$


Observe from Proposition 3.6 that when $b=1$, the smallest $d$ with $H_{3}\left(\mathcal{L}^{-}\right)_{-d} \neq(0)$ is $2 a$, while when $b \geq 2$, the smallest value is $2 a+1$. We assume henceforth that $b \geq 2$, as the case $b=1$ can be found in [BKM2] or can be gotten by modifying the argument below. Now from Propositions 1.9 and 3.6 we have

Corollary 3.7. For $a+1 \leq j \leq 2 a$,

$$
J_{-j} \cong \underbrace{V \otimes \cdots \otimes V}_{j-a-1 \text { times }} \otimes V(\nu)
$$

and

$$
J_{-(2 a+1)} \cong(\underbrace{V \otimes \cdots \otimes V}_{a \text { times }} \otimes V(\nu)) / V(\phi),
$$

where

$$
\begin{aligned}
& \nu=(a b+b-1) \epsilon_{1}+\epsilon_{2} \vdash b(a+1) \\
& \phi=(2 a b+b-2) \epsilon_{1}+\epsilon_{2}+\epsilon_{3} \vdash b(2 a+1) .
\end{aligned}
$$

From Corollary 3.7 we see that determining the structure of $J_{-j}$ for $j=$ $a+2, \ldots, 2 a+1$ involves knowing how to decompose the tensor product $V^{\otimes m}$ of $m=j-a-1$ copies of the representation $V$ into irreducible $G$-summands $V(\lambda)$ and then how to write $V(\lambda) \otimes V(\nu)$ as a sum of irreducible $G$-modules for $\lambda, \nu$ dominant weights. The first step in this analysis is the following

Proposition 3.10. Let $V$ be the $g \ell(n+1, \mathbb{C})$-module $V\left(b \Lambda_{1}\right)=V\left(b \epsilon_{1}\right)$. Then

$$
V^{\otimes m} \cong \sum_{\substack{\lambda \vdash m b \\ \ell(\lambda) \leq n+1, m}} K_{\lambda,\left\{b^{m}\right\}} V(\lambda)
$$

where $\left\{b^{m}\right\}$ denotes the partition of $m b$ having $m$ parts equal to $b$, and $K_{\lambda,\left\{b^{m}\right\}}$ is the Kostka number.

Proof. From the Littlewood-Richardson Rule (see for example, the discussion in $[\mathbf{M}]$, [BKM2], or [BBL], Chap. 7) we can derive the multiplicity of the irreducible summand $V(\lambda)$ in $\otimes^{m} V$ as follows: Let $\lambda_{1} \vdash b$ denote the partition of $b$ having just one part of size $b$. Associate to $\lambda_{1}$ its frame, which has just one row with $b$ boxes, and fill in those boxes with " 1 's". Append $b$ boxes to the frame of $\lambda_{1}$ in such a way that no two lie in the same column and the result is the frame of some partition $\lambda_{2} \vdash 2 b$. Fill in the adjoined 
boxes with "2's". Proceed in this fashion to arrive at a partition $\lambda=\lambda_{m}$ of $m b$ whose frame has been filled with $b$ "i's" for $i=1, \ldots, m$ in such a way that the numbers weakly increase across the rows and strictly increase down the columns. The result is a "semistandard tableau" of shape $\lambda$ where $\lambda \vdash m b$. The tableau's content is the partition $\left\{b^{m}\right\}$, as it contains $b$ "i's" for $i=1, \ldots, m$. The number of nonzero parts $\ell(\lambda)$ of $\lambda$, which is at most $m$ by the construction, must not exceed $n+1$. By the Littlewood-Richardson Rule, the multiplicity of $V(\lambda)$ in $\otimes^{m} V$ is the number of such semistandard tableaux, which is the Kostka number $K_{\lambda,\left\{b^{m}\right\}}$.

Theorem 3.11. Let $j$ be an integer such that $a+1 \leq j \leq 2 a+1$. Assume $\alpha=-\left(j \alpha_{0}+\sum_{i=1}^{n} k_{i} \alpha_{i}\right)=\left(j b-k_{1}\right) \epsilon_{1}+\left(k_{1}-k_{2}\right) \epsilon_{2}+\cdots+\left(k_{n-1}-k_{n}\right) \epsilon_{n}+k_{n} \epsilon_{n+1}$ is dominant so that $\alpha \vdash j b$. Then

$$
\begin{aligned}
& \operatorname{dim} J_{\alpha}^{-}=\operatorname{dim}\left(J_{-j}\right)_{\alpha} \\
& =\left\{\sum_{\substack{\pi \vdash j b \\
\ell(\pi) \leq n+1, j-a+1}}\left(\sum_{\substack{\lambda \vdash(j-a-1) b \\
\ell(\lambda) \leq n+1, j-a-1}} K_{\lambda,\left\{b^{j-a-1}\right\}} c_{\lambda, \nu}^{\pi}\right) K_{\pi, \alpha}\right\} \\
& -\delta_{2 a+1, j} K_{\phi, \alpha},
\end{aligned}
$$

where

$$
\begin{aligned}
& \nu=(a b+b-1) \epsilon_{1}+\epsilon_{2}=\{a b+b-1,1\} \vdash a b+b \\
& \phi=(2 a b+b-2) \epsilon_{1}+\epsilon_{2}+\epsilon_{3}=\left\{2 a b+b-2,1^{2}\right\} \vdash 2 a b+b .
\end{aligned}
$$

Proof. Corollary 3.7 gives

$$
J_{-j} \cong V^{\otimes^{j-a-1}} \otimes V(\nu)
$$

for $a+1 \leq j \leq 2 a$ and

$$
J_{-(2 a+1)} \cong\left(V^{\otimes^{a}} \otimes V(\nu)\right) / V(\phi)
$$

where $\nu=(a b+b-1) \epsilon_{1}+\epsilon_{2} \vdash a b+b$ and $\phi=(2 a b+b-2) \epsilon_{1}+\epsilon_{2}+\epsilon_{3} \vdash 2 a b+b$. Therefore by Proposition 3.10, we have

$$
J_{-j} \cong \sum_{\substack{\lambda \vdash(j-a-1) b \\ \ell(\lambda) \leq n+1, j-a-1}} K_{\lambda,\left\{b^{j-a-1}\right\}} V(\lambda) \otimes V(\nu),
$$

for $a+1 \leq j \leq 2 a$, and 


$$
J_{-(2 a+1)} \cong\left(\sum_{\substack{\lambda \vdash a b \\ \ell(\lambda) \leq n+1, a}} K_{\lambda,\left\{b^{a}\right\}} V(\lambda) \otimes V(\nu)\right) / V(\phi) .
$$

Now if $\lambda \vdash j-a-1$, then since $\nu$ has two nonzero parts, we have by the Littlewood-Richardson Rule,

$$
V(\lambda) \otimes V(\nu) \cong \sum_{\substack{\pi \vdash j b \\ \ell(\pi) \leq n+1, j-a+1}} c_{\lambda, \nu}^{\pi} V(\pi),
$$

where $c_{\lambda, \nu}^{\pi}$ is the Littlewood-Richardson coefficient (see [M] ). Since the multiplicity of $\alpha$ in $V(\pi)$ is the Kostka number $K_{\pi, \alpha}$, the assertions in (3.12) follow.

Remark. For $\lambda \vdash m$, the Kostka number $K_{\lambda,\left\{1^{m}\right\}}$ is just the number of standard tableaux (strictly increasing along each row and column) of shape $\lambda$ with entries in $\{1, \ldots, m\}$. That number equals $m ! / h(\lambda)$ where $h(\lambda)$ is the hook length of the partition $\lambda$. Thus, (3.12) in the case that $b=1$ is just (4.11) of [BKM2].

Now we need to compute the multiplicity $\operatorname{dim} \mathcal{F}_{\alpha}^{-}$of $\alpha$ in the free part. Recall that the weights of $V$ are

$$
W t=\left\{m_{1} \epsilon_{1}+\cdots+m_{n+1} \epsilon_{n+1} \mid m_{i} \geq 0 \text { and } m_{1}+\cdots+m_{n+1}=b\right\},
$$

and each weight has multiplicity one. As a consequence of Proposition 1.6 we have:

Proposition 3.13. Assume $\alpha=-\left(j \alpha_{0}+\sum_{i=1}^{n} k_{i} \alpha_{i}\right)=\left(j b-k_{1}\right) \epsilon_{1}+\left(k_{1}-\right.$ $\left.k_{2}\right) \epsilon_{2}+\cdots+\left(k_{n-1}-k_{n}\right) \epsilon_{n}+k_{n} \epsilon_{n+1}$ is dominant so that $\alpha \vdash j b$. Let

$$
W t=\left\{m_{1} \epsilon_{1}+\cdots+m_{n+1} \epsilon_{n+1} \mid m_{i} \geq 0 \text { and } m_{1}+\cdots+m_{n+1}=b\right\} .
$$

denote the set of weights of $V\left(b \epsilon_{1}\right)$, and let $W t=\left\{\omega_{1}, \omega_{2}, \ldots\right\}$ be an enumeration of those weights. Then

$$
\operatorname{dim} \mathcal{F}_{\alpha}^{-}=\sum_{\omega \mid \alpha} \mu\left(\frac{\alpha}{\omega}\right) \frac{\omega}{\alpha} D(\omega)
$$

where $\mu$ denotes the classical Möbius function; $\omega \mid \alpha$ if $\alpha=\kappa \omega$ for some 
positive integer $\kappa$, in which case $\frac{\alpha}{\omega}=\kappa$ and $\frac{\omega}{\alpha}=\frac{1}{\kappa} ;$ and

$$
D(\omega)=\sum_{(t) \in T(\omega)} \frac{\left(\left(\sum_{i} t_{i}\right)-1\right) !}{\prod_{i}\left(t_{i} !\right)}
$$

where $T(\omega)=\left\{(t)=\left(t_{1}, t_{2}, \ldots\right) \mid t_{i} \in \mathbb{Z}^{\geq 0}\right.$ and $\left.\sum_{i} t_{i} \omega_{i}=\omega\right\}$.

We close this section by applying the multiplicity formulas derived in this section to calculate root multiplicities for the algebras $I A_{n}(2,2)$ with $n \geq 3$. In particular, for $I A_{3}(2,2)$ we compute the multiplicities of the dominant roots $\alpha=-\left(j \alpha_{0}+k_{1} \alpha_{1}+k_{2} \alpha_{2}+k_{3} \alpha_{3}\right)=\theta_{1} \epsilon_{1}+\theta_{2} \epsilon_{2}+\theta_{3} \epsilon_{3}+\theta_{4} \epsilon_{4}$ where $j=2,3,4,5=2 a+1$. We explicitly exhibit the calculations for one choice of $\alpha$ for each value $j=3,4,5$ and then display the remainder in the tables below. It follows from ([BKM2], Prop. 4.12) that the multiplicity of such roots $\alpha$ is the same for all algebras $I A_{n}(2,2)$ with $n \geq 3$.

To avoid cumbersome notation we adopt the shorthand for the dominant root which illustrates its parts as a partition. Thus, we write $\left\{4,1^{2}\right\}$ for the root $\alpha=-\left(3 \alpha_{0}+2 \alpha_{1}+\alpha_{2}\right)=4 \epsilon_{1}+\epsilon_{2}+\epsilon_{3}$. For the algebra $I A_{3}(2,2)$ we have $V=V\left(2 \epsilon_{1}\right)=V(\{2\})$ and $\alpha_{0}=-2 \epsilon_{1}$, and the weights of $V$ are

$$
W t=\left\{2 \epsilon_{1}, \epsilon_{1}+\epsilon_{2}, 2 \epsilon_{2}, \epsilon_{1}+\epsilon_{3}, \epsilon_{2}+\epsilon_{3}, 2 \epsilon_{3}, \epsilon_{1}+\epsilon_{4}, \epsilon_{2}+\epsilon_{4}, \epsilon_{3}+\epsilon_{4}, 2 \epsilon_{4}\right\} .
$$

Recall by (3.3) that $J^{-}$is generated by $J_{-3} \cong V(\nu)=V\left(5 \epsilon_{1}+\epsilon_{2}\right)=$ $V(\{5,1\})$. Then by (3.8) and (3.9) and the Littlewood-Richardson rule (see [BKM2] or [BBL], Chap. 7),

$$
\begin{aligned}
J_{-4} \cong V & \otimes V(\nu)=V(\{2\}) \otimes V(\{5,1\}) \\
\cong & V(\{7,1\}) \oplus V(\{6,2\}) \oplus V\left(\left\{6,1^{2}\right\}\right) \oplus V(\{5,3\}) \oplus V(\{5,2,1\}) \\
J_{-5} \cong(V & \otimes V \otimes V(\nu)) / V(\phi) \\
& =(V(\{2\}) \otimes V(\{2\}) \otimes V(\{5,1\})) / V\left(\left\{8,1^{2}\right\}\right) \\
& \cong V(\{9,1\}) \oplus 2 V(\{8,2\}) \oplus V\left(\left\{8,1^{2}\right\}\right) \\
& \oplus 3 V(\{7,3\}) \oplus 4 V(\{7,2,1\}) \oplus V\left(\left\{7,1^{3}\right\}\right) \\
& \oplus 2 V(\{6,4\}) \oplus 4 V(\{6,3,1\}) \\
& \oplus 2 V\left(\left\{6,2^{2}\right\}\right) \oplus 2 V\left(\left\{6,2,1^{2}\right\}\right) \oplus V(\{5,5\}) \\
& \oplus 2 V(\{5,4,1\}) \oplus 2 V(\{5,3,2\}) \oplus V\left(\left\{5,3,1^{2}\right\}\right) \oplus V\left(\left\{5,2^{2}, 1\right\}\right) .
\end{aligned}
$$


Example 1. Consider the root $\alpha=-\left(3 \alpha_{0}+2 \alpha_{1}+\alpha_{2}\right)=4 \epsilon_{1}+\epsilon_{2}+\epsilon_{3}=\left\{4,1^{2}\right\}$ for $I A_{3}(2,2)$. Then

$$
\begin{aligned}
\alpha & =4 \epsilon_{1}+\epsilon_{2}+\epsilon_{3} \\
& =\left(2 \epsilon_{1}\right)+\left(2 \epsilon_{1}\right)+\left(\epsilon_{2}+\epsilon_{3}\right) \\
& =\left(2 \epsilon_{1}\right)+\left(\epsilon_{1}+\epsilon_{2}\right)+\left(\epsilon_{1}+\epsilon_{3}\right)
\end{aligned}
$$

so that by (3.14)

$$
\operatorname{dim} \mathcal{F}_{\alpha}^{-}=\frac{2 !}{2 !}+\frac{2 !}{1 !}=3
$$

Now $\operatorname{dim} J_{\alpha}^{-}=\operatorname{dim}\left(J_{-3}\right)_{\alpha}=\operatorname{dim} V(\{5,1\})_{\alpha}=K_{\{5,1\}, \alpha}=2$ by Table 9.12 of [BBL]. Therefore $\operatorname{dim} \mathcal{L}_{\alpha}=3-2=1$.

Example 2. Let $\alpha=-\left(4 \alpha_{0}+3 \alpha_{1}+\alpha_{2}\right)=5 \epsilon_{1}+2 \epsilon_{2}+\epsilon_{3}=\{5,2,1\}$ for $I A_{3}(2,2)$. Then

$$
\begin{aligned}
\alpha & =5 \epsilon_{1}+2 \epsilon_{2}+\epsilon_{3} \\
& =\left(2 \epsilon_{1}\right)+\left(2 \epsilon_{1}\right)+\left(\epsilon_{1}+\epsilon_{2}\right)+\left(\epsilon_{2}+\epsilon_{3}\right) \\
& =\left(2 \epsilon_{1}\right)+\left(2 \epsilon_{1}\right)+\left(2 \epsilon_{2}\right)+\left(\epsilon_{1}+\epsilon_{3}\right) \\
& =\left(2 \epsilon_{1}\right)+\left(\epsilon_{1}+\epsilon_{2}\right)+\left(\epsilon_{1}+\epsilon_{2}\right)+\left(\epsilon_{1}+\epsilon_{3}\right)
\end{aligned}
$$

and by (3.14)

$$
\operatorname{dim} \mathcal{F}_{\alpha}^{-}=\frac{3 !}{2 !}+\frac{3 !}{2 !}+\frac{3 !}{2 !}=9
$$

Since $\operatorname{dim} J_{\alpha}^{-}=\operatorname{dim}\left(J_{-4}\right)_{\alpha}=K_{\{7,1\}, \alpha}+K_{\{6,2\}, \alpha}+K_{\left\{6,1^{2}\right\}, \alpha}+K_{\{5,3\}, \alpha}+$ $K_{\{5,2,1\}, \alpha}=2+2+1+1+1$, we have $\operatorname{dim} \mathcal{L}_{\alpha}=9-7=2$. (We have used the fact (see for example [S], Chap. 2) that $K_{\pi, \alpha}$ represents the number of semistandard tableaux of shape $\pi$ and content $\alpha$ to evaluate $K_{\pi, \alpha}$.)

Example 3. In this final example assume $\alpha=-\left(5 \alpha_{0}+4 \alpha_{1}+\alpha_{2}\right)=6 \epsilon_{1}+$ $3 \epsilon_{2}+\epsilon_{3}=\{6,3,1\}$ for $I A_{3}(2,2)$. Then

$$
\begin{aligned}
\alpha & =6 \epsilon_{1}+3 \epsilon_{2}+\epsilon_{3} \\
& =\left(2 \epsilon_{1}\right)+\left(2 \epsilon_{1}\right)+\left(2 \epsilon_{1}\right)+\left(2 \epsilon_{2}\right)+\left(\epsilon_{2}+\epsilon_{3}\right) \\
& =\left(2 \epsilon_{1}\right)+\left(2 \epsilon_{1}\right)+\left(\epsilon_{1}+\epsilon_{2}\right)+\left(\epsilon_{1}+\epsilon_{2}\right)+\left(\epsilon_{2}+\epsilon_{3}\right) \\
& =\left(2 \epsilon_{1}\right)+\left(2 \epsilon_{1}\right)+\left(\epsilon_{1}+\epsilon_{2}\right)+\left(2 \epsilon_{2}\right)+\left(\epsilon_{1}+\epsilon_{3}\right) \\
& =\left(2 \epsilon_{1}\right)+\left(\epsilon_{1}+\epsilon_{2}\right)+\left(\epsilon_{1}+\epsilon_{2}\right)+\left(\epsilon_{1}+\epsilon_{2}\right)+\left(\epsilon_{1}+\epsilon_{3}\right)
\end{aligned}
$$


and from (3.14) we obtain,

$$
\operatorname{dim} \mathcal{F}_{\alpha}^{-}=\frac{4 !}{3 !}+\frac{4 !}{2 ! 2 !}+\frac{4 !}{2 !}+\frac{4 !}{3 !}=26 .
$$

By our calculations above

$$
\begin{aligned}
\operatorname{dim} J_{\alpha}^{-}=\operatorname{dim}\left(J_{-5}\right)_{\alpha} & =K_{\{9,1\}, \alpha}+2 K_{\{8,2\}, \alpha}+K_{\left\{8,1^{2}\right\}, \alpha}+3 K_{\{7,3\}, \alpha} \\
& +4 K_{\{7,2,1\}, \alpha}+K_{\left\{7,1^{3}\right\}, \alpha}+2 K_{\{6,4\}, \alpha}+4 K_{\{6,3,1\}, \alpha} \\
& +2 K_{\left\{6,2^{2}\right\}, \alpha}+2 K_{\left\{6,2,1^{2}\right\}, \alpha}+K_{\{5,5\}, \alpha}+2 K_{\{5,4,1\}, \alpha} \\
& +2 K_{\{5,3,2\}, \alpha}+K_{\left\{5,3,1^{2}\right\}, \alpha}+K_{\left\{5,2^{2}, 1\right\}, \alpha} \\
& =2+4+1+6+4+0+2+4 \\
& +0+0+0+0+0+0+0 \\
& =23
\end{aligned}
$$

so that $\operatorname{dim} \mathcal{L}_{\alpha}=26-23=3$.

Root Multiplicities in $I A_{n}(2,2)$ for $n \geq 3$.

\begin{tabular}{|r|c|c|c|c|}
\hline $\begin{array}{c}\text { Dominant } \\
\text { Roots: } \alpha\end{array}$ & $\operatorname{deg}(\alpha)$ & $\operatorname{dim} \mathcal{F}_{\alpha}^{-}$ & $\operatorname{dim} J_{\alpha}^{-}$ & $\operatorname{dim} \mathcal{L}_{\alpha}^{-}$ \\
\hline$\{3,1\}$ & -2 & 1 & 0 & 1 \\
$\left\{2^{2}\right\}$ & -2 & 1 & 0 & 1 \\
$\left\{2,1^{2}\right\}$ & -2 & 2 & 0 & 2 \\
$\left\{1^{4}\right\}$ & -2 & 3 & 0 & 3 \\
$\{4,2\}$ & -3 & 2 & 1 & 1 \\
$\left\{3^{2}\right\}$ & -3 & 2 & 1 & 1 \\
$\left\{4,1^{2}\right\}$ & -3 & 3 & 2 & 1 \\
$\{3,2,1\}$ & -3 & 5 & 2 & 3 \\
$\left\{2^{3}\right\}$ & -3 & 7 & 2 & 5 \\
$\left\{3,1^{3}\right\}$ & -3 & 8 & 3 & 5 \\
$\left\{2^{2}, 1^{2}\right\}$ & -3 & 11 & 3 & 8 \\
$\{5,3\}$ & -4 & 4 & 3 & 1 \\
\hline
\end{tabular}


Root Multiplicities in $I A_{n}(2,2)$ for $n \geq 3$.

\begin{tabular}{|r|c|c|c|c|}
\hline Dominant & & & & \\
Roots: $\alpha$ & $\operatorname{deg}(\alpha)$ & $\operatorname{dim} \mathcal{F}_{\alpha}^{-}$ & $\operatorname{dim} J_{\alpha}^{-}$ & $\operatorname{dim} \mathcal{L}_{\alpha}^{-}$ \\
$\{5,2,1\}$ & -4 & 4 & 3 & 1 \\
$\{4,3,1\}$ & -4 & 9 & 7 & 2 \\
$\left\{4,2^{2}\right\}$ & -4 & 13 & 8 & 5 \\
$\left\{3^{2}, 2\right\}$ & -4 & 22 & 11 & 11 \\
$\left\{5,1^{3}\right\}$ & -4 & 15 & 12 & 3 \\
$\left\{4,2,1^{2}\right\}$ & -4 & 30 & 17 & 13 \\
$\left\{3^{2}, 1^{2}\right\}$ & -4 & 31 & 18 & 13 \\
$\left\{3,2^{2}, 1\right\}$ & -4 & 48 & 22 & 26 \\
$\left\{2^{4}\right\}$ & -4 & 69 & 27 & 42 \\
$\{6,4\}$ & -5 & 9 & 8 & 1 \\
$\left\{5^{2}\right\}$ & -5 & 10 & 9 & 1 \\
$\{6,3,1\}$ & -5 & 26 & 23 & 3 \\
$\left\{6,2^{2}\right\}$ & -5 & 36 & 31 & 5 \\
$\{5,4,1\}$ & -5 & 35 & 28 & 7 \\
$\{5,3,2\}$ & -5 & 60 & 45 & 15 \\
$\left\{4^{2}, 2\right\}$ & -5 & 71 & 49 & 22 \\
$\left\{4,3^{2}\right\}$ & -5 & 88 & 58 & 30 \\
$\left\{6,2,1^{2}\right\}$ & -5 & 62 & 42 & 20 \\
$\left\{5,3,1^{2}\right\}$ & -5 & 104 & 77 & 27 \\
$\left\{5,2^{2}, 1\right\}$ & -5 & 138 & 103 & 35 \\
$\left\{4^{2}, 1^{2}\right\}$ & -5 & 111 & 84 & 27 \\
$\{4,3,2,1\}$ & -5 & 158 & 131 & 27 \\
$\left\{4,2^{3}\right\}$ & -5 & 282 & 169 & 113 \\
\hline 2 & -5 & 240 & 150 & 90 \\
\hline 3 & 366 & 195 & 171 \\
\hline
\end{tabular}


Remarks. Any partition in the table having only 2 nonzero parts corresponds to a root $-\left(k_{0} \alpha_{0}+k_{1} \alpha_{1}\right)$, which is a root for all algebras $I A_{n}(2,2)$ with $n \geq 1$. By ([BKM2], Prop. 4.12) its multiplicity in $I A_{n}(2,2)$ for all $n \geq 1$ is the same as its multiplicity in $I A_{1}(2,2)$. The algebra $I A_{1}(2,2)$ is just the affine algebra $A_{1}^{(1)}$ and all roots for $A_{1}^{(1)}$ have multiplicity one ([Kac], Cor. 7.4), so all partitions with 2 nonzero parts have multiplicity one. Any partition $\theta=\left\{\theta_{1} \geq \theta_{2} \geq \theta_{3}>0\right\}$ with 3 nonzero parts corresponds to a root $-\left(k_{0} \alpha_{0}+k_{1} \alpha_{1}+k_{2} \alpha_{2}\right)$, where $\theta_{1}=2 k_{0}-k_{1}, \theta_{2}=k_{1}-k_{2}$ and $\theta_{3}=k_{2}$. The multiplicity of $\theta$ in $I A_{n}(2,2)$ for $n \geq 2$ is the same as in $I A_{2}(2,2)$, which is the hyperbolic Kac-Moody algebra $H A_{1}^{(1)}$ studied by Feingold and Frenkel [FF] and by Kang [Kan1] [Kan2]. Assume

$$
\Gamma(\alpha)=\left(\begin{array}{lc}
k_{1}-k_{2} & k_{1}-k_{0} \\
k_{1}-k_{0} & k_{2}
\end{array}\right)=\left(\begin{array}{cc}
\theta_{2} & 1 / 2\left(\theta_{2}+\theta_{3}-\theta_{1}\right) \\
1 / 2\left(\theta_{2}+\theta_{3}-\theta_{1}\right) & \theta_{3}
\end{array}\right) .
$$

Then whenever $\alpha$ is conjugate under the Weyl group to a root having $\alpha_{2}$ coefficient equal to -1 or 1 , its multiplicity is given by $p(\operatorname{det}(\Gamma(\alpha))+1)$ where $p(\cdot)$ is the classical partition function (see $[\mathbf{F F}]$, p. 117). All the partitions with 3 nonzero parts in the table have such expressions for their multiplicities.

\section{Root Multiplicity Formulas.}

In this final section we apply the Euler-Poincaré Principle (see [CE]) and the extension of Kostant's formula given in [GL] to derive two root multiplicity formulas, one of which is recursive and the other closed form, for the algebra $I A_{n}(a, b) \cong \mathcal{L}=\mathcal{L}\left(G, V, V^{*}, \psi\right)$. Since $\operatorname{dim} \mathcal{L}_{\alpha}=\operatorname{dim} \mathcal{L}_{-\alpha}$ for any root $\alpha$, it suffices to determine $\operatorname{dim} \mathcal{L}_{\alpha}=\operatorname{dim} \mathcal{L}_{\alpha}^{-}$for $\alpha=-\left(j \alpha_{0}+\sum_{i=1}^{n} k_{i} \alpha_{i}\right)$. Needed in the argument is the construction of the homology $H_{*}\left(\mathcal{L}^{-}\right)=H_{*}\left(\mathcal{L}^{-}, \mathbb{C}\right)$ with coefficients in the trivial $\mathcal{L}^{-}$-module $\mathbb{C}$.

Consider the complex $\left(\wedge^{*}\left(\mathcal{L}^{-}\right), d_{*}\right)$ :

$$
\begin{aligned}
& \cdots \longrightarrow \wedge^{k+1}\left(\mathcal{L}^{-}\right) \stackrel{d_{k+1}}{\longrightarrow} \wedge^{k}\left(\mathcal{L}^{-}\right) \stackrel{d_{k}}{\longrightarrow} \wedge^{k-1}\left(\mathcal{L}^{-}\right) \stackrel{d_{k-1}}{\longrightarrow} \cdots \\
& \stackrel{d_{2}}{\longrightarrow} \wedge^{1}\left(\mathcal{L}^{-}\right) \stackrel{d_{1}}{\longrightarrow} \wedge^{0}\left(\mathcal{L}^{-}\right) \stackrel{d_{0}}{\longrightarrow} \mathbb{C} \longrightarrow(0)
\end{aligned}
$$

where $d_{k}$ is defined by 


$$
\begin{aligned}
d_{k}\left(x_{1} \wedge\right. & \left.x_{2} \wedge \cdots \wedge x_{k}\right) \\
& =\sum_{1 \leq s<t \leq k}(-1)^{s+t-1}\left(\left[x_{s}, x_{t}\right] \wedge x_{1} \wedge \cdots \wedge \widehat{x}_{s} \wedge \cdots \wedge \widehat{x}_{t} \wedge \cdots \wedge x_{k}\right)
\end{aligned}
$$

Then for all $k \geq 0, H_{k}\left(\mathcal{L}^{-}\right)=\left(\operatorname{Ker} d_{k}\right) /\left(\operatorname{Im} d_{k+1}\right)$.

By the Euler-Poincaré Principle we have,

$$
\sum_{k=0}^{\infty}(-1)^{k} \operatorname{ch}\left(\wedge^{k}\left(\mathcal{L}^{-}\right)\right)=\sum_{k=0}^{\infty}(-1)^{k} \operatorname{ch}\left(H_{k}\left(\mathcal{L}^{-}\right)\right)
$$

where for any completely reducible $G$-module $M=\oplus \sum_{\lambda \in \mathcal{H}^{*}} M_{\lambda}$ the formal character is, by definition, the sum

$$
\operatorname{ch}(M)=\sum_{\lambda \in \mathcal{H}^{*}}\left(\operatorname{dim} M_{\lambda}\right) e(\lambda)
$$

If $\alpha=-\left(j \alpha_{0}+\sum_{i=1}^{n} k_{i} \alpha_{i}\right)$ is a root with $j \geq 2$, (the case $j=1$ corresponds to the weights of $V$ which have multiplicity one), then since $H_{0}\left(\mathcal{L}^{-}\right) \cong \mathbb{C}$ and $H_{1}\left(\mathcal{L}^{-}\right) \cong \mathcal{L}^{-} /\left[\mathcal{L}^{-}, \mathcal{L}^{-}\right] \cong \mathcal{L}_{-1}=V$, it follows from (4.1) that

$$
\begin{aligned}
\operatorname{dim} \mathcal{L}_{\alpha}^{-} & =\operatorname{dim}\left(\mathcal{L}_{-j}\right)_{\alpha} \\
& =\sum_{k=2}^{\infty}(-1)^{k} \operatorname{dim} \wedge^{k}\left(\mathcal{L}^{-}\right)_{\alpha}-\sum_{k=2}^{\infty}(-1)^{k} \operatorname{dim} H_{k}\left(\mathcal{L}^{-}\right)_{\alpha} .
\end{aligned}
$$

If $k>j$, any weight of $\wedge^{k}\left(\mathcal{L}^{-}\right)$has degree $\leq-k<-j$. Hence $\operatorname{dim} \wedge^{k}\left(\mathcal{L}^{-}\right)_{\alpha}=0$, as $\operatorname{deg} \alpha=-j$. Therefore for $k>j, \operatorname{dim} H_{k}\left(\mathcal{L}^{-}\right)_{\alpha}=$ $\operatorname{dim}\left(\operatorname{Ker} d_{k}\right)_{\alpha}-\operatorname{dim}\left(\operatorname{Im} d_{k+1}\right)_{\alpha}=0$. Consequently, the sum on the right hand side of (4.2) reduces to

$$
\operatorname{dim} \mathcal{L}_{\alpha}^{-}=\sum_{k=2}^{j}(-1)^{k} \operatorname{dim} \wedge^{k}\left(\mathcal{L}^{-}\right)_{\alpha}-\sum_{k=2}^{j}(-1)^{k} \operatorname{dim} H_{k}\left(\mathcal{L}^{-}\right)_{\alpha} .
$$

Kostant's formula (3.5) gives

$$
\operatorname{dim} H_{k}\left(\mathcal{L}^{-}\right)_{\alpha}=\sum_{\substack{w \in W^{\prime} \\ l(w)=k}} \operatorname{dim} V(w \rho-\rho)_{\alpha}
$$


where $V(w \rho-\rho)_{\alpha}=0$ unless $\operatorname{deg}(w \rho-\rho)=-j=\operatorname{deg}(\alpha)$. If $\operatorname{deg}(w \rho-\rho)=$ $-j$, then $\operatorname{dim} V(w \rho-\rho)_{\alpha}=\operatorname{dim} V(w \rho-\rho)_{\bar{\alpha}}=K_{w \rho-\rho, \bar{\alpha}}$, where $\bar{\alpha}$ is the unique dominant weight conjugate to $\alpha$, and $K_{w \rho-\rho, \bar{\alpha}}$ is the Kostka number. For convenience, let $K_{w \rho-\rho, \alpha}=\operatorname{dim} V(w \rho-\rho)_{\alpha}=K_{w \rho-\rho, \bar{\alpha}}$. Thus,

$$
\operatorname{dim} H_{k}\left(\mathcal{L}^{-}\right)_{\alpha}=\sum_{\begin{array}{c}
w \in W^{\prime} \\
\boldsymbol{w}(w)=k \\
\operatorname{deg}(w \rho-\rho)=-j
\end{array}} K_{w \rho-\rho, \alpha} .
$$

We also make use of the following total order defined on the root lattice of $\mathcal{L}^{-}$. If $\alpha=\theta_{1} \epsilon_{1}+\cdots+\theta_{n+1} \epsilon_{n+1}$ and $\beta=\zeta_{1} \epsilon_{1}+\cdots+\zeta_{n+1} \epsilon_{n+1}$, then we say $\alpha<\beta$ iff $\sum_{i=1}^{n+1} \theta_{i}<\sum_{i=1}^{n+1} \zeta_{i}$ or $\sum_{i=1}^{n+1} \theta_{i}=\sum_{i=1}^{n+1} \zeta_{i}$ and $\theta_{i}<\zeta_{i}$ for some $i$ and $\theta_{s}=\zeta_{s}$ for $i<s \leq n+1$. Then equations (4.3) and (4.4) combine to give the following recursive formula for the root multiplicities.

Theorem 4.5. Let $I A_{n}(a, b) \cong \mathcal{L}=\mathcal{L}\left(G, V, V^{*}, \psi\right)$, and for any root $\beta$ of $\mathcal{L}^{-}$, let $m_{\beta}=\operatorname{dim} \mathcal{L}_{\beta}^{-}$. If $\alpha=-\left(j \alpha_{0}+\sum_{i=1}^{n} k_{i} \alpha_{i}\right)$ for some $j \geq 2$, then

$$
\begin{gathered}
\operatorname{dim} \mathcal{L}_{\alpha}=\operatorname{dim} \mathcal{L}_{\alpha}^{-}=\sum_{k=2}^{j}(-1)^{k} \sum_{\substack{\beta_{1}<\ldots<\beta_{r} \\
p_{1}+\ldots+p_{r}=k \\
p_{1} \beta_{1}+\cdots+p_{r} \beta_{r}=\alpha}}\left(\begin{array}{c}
m_{\beta_{1}} \\
p_{1}
\end{array}\right) \cdots\left(\begin{array}{c}
m_{\beta_{r}} \\
p_{r}
\end{array}\right) \\
-\sum_{k=2}^{j}(-1)^{k} \sum_{\substack{w \in W^{\prime} \\
l(w)=k \\
\operatorname{deg}(w \rho-\rho)=-j}} K_{w \rho-\rho, \alpha} .
\end{gathered}
$$

Next we present an example to illustrate how this recursive formula can be applied in an actual root multiplicity computation.

Example 4.6. Once again we assume the algebra is of type $I A_{3}(2,2)$, and we let $\alpha=-\left(6 \alpha_{0}+6 \alpha_{1}+2 \alpha_{2}+\alpha_{3}\right)=6 \epsilon_{1}+4 \epsilon_{2}+\epsilon_{3}+\epsilon_{4}=\left\{6,4,1^{2}\right\}$. (This is a root whose multiplicity cannot be computed using the results of Section 3 because its degree $-j=-6$ satisfies $j>2 a+1=5$.) Then by (4.3),

$$
\begin{aligned}
\operatorname{dim} \mathcal{L}_{\alpha}= & \operatorname{dim} \mathcal{L}_{\alpha}^{-} \\
= & \operatorname{dim} \wedge^{2}\left(\mathcal{L}^{-}\right)_{\alpha}-\operatorname{dim} \wedge^{3}\left(\mathcal{L}^{-}\right)_{\alpha}+\operatorname{dim} \wedge^{4}\left(\mathcal{L}^{-}\right)_{\alpha}-\operatorname{dim} \wedge^{5}\left(\mathcal{L}^{-}\right)_{\alpha} \\
& +\operatorname{dim} \wedge^{6}\left(\mathcal{L}^{-}\right)_{\alpha}-\operatorname{dim} H_{2}\left(\mathcal{L}^{-}\right)_{\alpha}+\operatorname{dim} H_{3}\left(\mathcal{L}^{-}\right)_{\alpha} \\
& -\operatorname{dim} H_{4}\left(\mathcal{L}^{-}\right)_{\alpha}+\operatorname{dim} H_{5}\left(\mathcal{L}^{-}\right)_{\alpha}-\operatorname{dim} H_{6}\left(\mathcal{L}^{-}\right)_{\alpha} .
\end{aligned}
$$


Now $\operatorname{dim} \wedge^{k}\left(\mathcal{L}^{-}\right)_{\alpha}=\operatorname{dim}\left\{x_{1} \wedge x_{2} \wedge \cdots \wedge x_{k} \mid x_{i} \in \mathcal{L}_{-r_{i}}, r_{1}+\cdots+r_{k}=\right.$ $\left.6 ; w t\left(x_{1}\right)+\cdots+w t\left(x_{k}\right)=\alpha\right\}$, and we can use the tables in the previous section to compute these dimensions. As a result we obtain

$$
\begin{aligned}
& \operatorname{dim} \wedge^{2}\left(\mathcal{L}^{-}\right)_{\alpha}=225 \\
& \operatorname{dim} \wedge^{3}\left(\mathcal{L}^{-}\right)_{\alpha}=251 \\
& \operatorname{dim} \wedge^{4}\left(\mathcal{L}^{-}\right)_{\alpha}=102 \\
& \operatorname{dim} \wedge^{5}\left(\mathcal{L}^{-}\right)_{\alpha}=15 \\
& \operatorname{dim} \wedge^{6}\left(\mathcal{L}^{-}\right)_{\alpha}=0 .
\end{aligned}
$$

For the homology portion, Kostant's formula (3.5) and Lemma 3.4 give:

$$
\begin{aligned}
& H_{2}\left(\mathcal{L}^{-}\right)=\sum_{\substack{w \in W^{\prime} \\
l(w)=2}} V(w \rho-\rho)=V\left(s_{0} s_{1} \rho-\rho\right) \\
& =V\left(-3 \alpha_{0}-\alpha_{1}\right)=V(\{5,1\}) \\
& H_{3}\left(\mathcal{L}^{-}\right)=\sum_{\substack{w \in W^{\prime} \\
l(w)=3}} V(w \rho-\rho)=V\left(s_{0} s_{1} s_{0} \rho-\rho\right) \oplus V\left(s_{0} s_{1} s_{2} \rho-\rho\right) \\
& =V\left(-6 \alpha_{0}-3 \alpha_{1}\right) \oplus V\left(-5 \alpha_{0}-2 \alpha_{1}-\alpha_{2}\right) \\
& =V(\{9,3\}) \oplus V\left(\left\{8,1^{2}\right\}\right) \\
& H_{4}\left(\mathcal{L}^{-}\right)=\sum_{\substack{w \in W^{\prime} \\
l(w)=4}} V(w \rho-\rho)=V\left(\left(s_{0} s_{1}\right)^{2} \rho-\rho\right) \oplus V\left(s_{0} s_{1} s_{2} s_{3} \rho-\rho\right) \\
& =V\left(-10 \alpha_{0}-6 \alpha_{1}\right) \oplus V\left(-7 \alpha_{0}-3 \alpha_{1}-2 \alpha_{2}-\alpha_{3}\right) \\
& =V(\{14,6\}) \oplus V\left(\left\{11,1^{3}\right\}\right) \\
& H_{5}\left(\mathcal{L}^{-}\right)=\sum_{\substack{w \in W^{\prime} \\
l(w)=5}} V(w \rho-\rho)=V\left(\left(s_{0} s_{1}\right)^{2} s_{0} \rho-\rho\right) \oplus V\left(s_{0} s_{1} s_{0} s_{1} s_{2} \rho-\rho\right) \\
& =V\left(-15 \alpha_{0}-10 \alpha_{1}\right) \oplus V\left(-8 \alpha_{0}-4 \alpha_{1}-\alpha_{2}\right) \\
& =V(\{20,10\}) \oplus V(\{12,3,1\}) \\
& H_{6}\left(\mathcal{L}^{-}\right)=\sum_{\substack{w \in W^{\prime} \\
l(w)=6}} V(w \rho-\rho) \\
& =V\left(\left(s_{0} s_{1}\right)^{3} \rho-\rho\right) \oplus V\left(\left(s_{0} s_{1}\right)^{2} s_{2} s_{3} \rho-\rho\right) \oplus V\left(\left(s_{0} s_{1}\right)^{2} s_{2} s_{1} \rho-\rho\right) \\
& =V\left(-21 \alpha_{0}-15 \alpha_{1}\right) \oplus V\left(-18 \alpha_{0}-12 \alpha_{1}-2 \alpha_{2}-\alpha_{3}\right) \\
& \oplus V\left(-18 \alpha_{0}-11 \alpha_{1}-2 \alpha_{2}\right) \\
& =V(\{27,15\}) \oplus V\left(\left\{24,10,1^{2}\right\}\right) \oplus V(\{25,9,2\}) \text {. }
\end{aligned}
$$


From this we see that $\operatorname{dim} H_{k}\left(\mathcal{L}^{-}\right)_{\alpha}=0$ except when $k=3$ where

$$
\operatorname{dim} H_{3}\left(\mathcal{L}^{-}\right)_{\alpha}=\operatorname{dim} V(\{9,3\})_{\alpha}=K_{\{9,3\}, \alpha}=4 .
$$

Thus,

$$
\operatorname{dim} \mathcal{L}_{\alpha}=\operatorname{dim} \mathcal{L}_{\alpha}^{-}=225-251+102-15+4=65 .
$$

With the Euler-Poincaré Principle as a guide, we define $M$ to be the following formal alternating direct sum of finite dimensional modules for $G=g \ell(n+1, \mathbb{C})$ :

$$
\begin{aligned}
M & =\sum_{k=1}^{\infty}(-1)^{k+1} H_{k}\left(\mathcal{L}^{-}\right) \\
& =\sum_{k=1}^{\infty}(-1)^{k+1} \sum_{\substack{w \in W^{\prime} \\
l(w)=k}} V(w \rho-\rho) \\
& =\sum_{w \in W^{\prime}}(-1)^{l(w)+1} V(w \rho-\rho) .
\end{aligned}
$$

Then for $\alpha=-\left(j \alpha_{0}+\sum_{i=1}^{n} k_{i} \alpha_{i}\right)$, we set

$$
\operatorname{dim} M_{\alpha}=\sum_{\substack{w \in W^{\prime} \\ l(w) \leq j \\ \operatorname{deg}(w \rho-\rho)=-j}}(-1)^{l(w)+1} K_{w \rho-\rho, \alpha},
$$

and define the formal character of $M$ to be

$$
\operatorname{ch}(M)=\sum_{\alpha}\left(\operatorname{dim} M_{\alpha}\right) e(\alpha)
$$

It may be that $\operatorname{dim} M_{\alpha}$ is nonpositive for certain values of $\alpha$. Let $\tau_{1}, \tau_{2}, \ldots$ be an enumeration of the weights in $\left\{\alpha \mid \operatorname{dim} M_{\alpha} \neq 0\right\}$ compatible with the total ordering on the root lattice of $\mathcal{L}^{-}$given above, and for $\tau$ define

$$
T(\tau)=\left\{\underline{t}=\left(t_{1}, t_{2}, \ldots\right) \mid t_{i} \in \mathbb{Z}^{\geq 0}, \quad \sum_{i} t_{i} \tau_{i}=\tau\right\}
$$

and

$$
B(\tau)=\sum_{\underline{t} \in T(\tau)} \frac{\left(\left(\sum_{i} t_{i}\right)-1\right) !}{\prod_{i}\left(t_{i} !\right)} \prod_{i}\left(\operatorname{dim} M_{\tau_{i}}\right)^{t_{i}} .
$$


Then we have

Theorem 4.12. For any root $\alpha=-\left(j \alpha_{0}+\sum_{i=1}^{n} k_{i} \alpha_{i}\right)$ of $I A_{n}(a, b) \cong \mathcal{L}=$ $\mathcal{L}\left(G, V, V^{*}, \psi\right)$,

$$
\begin{aligned}
\operatorname{dim} \mathcal{L}_{\alpha}=\operatorname{dim} \mathcal{L}_{\alpha}^{-} & =\sum_{\tau \mid \alpha} \mu\left(\frac{\alpha}{\tau}\right) \frac{\tau}{\alpha} B(\tau) \\
& =\sum_{\tau \mid \alpha} \mu\left(\frac{\alpha}{\tau}\right) \frac{\tau}{\alpha} \sum_{\underline{t} \in T(\tau)} \frac{\left(\left(\sum_{i} t_{i}\right)-1\right) !}{\prod_{i}\left(t_{i} !\right)} \\
& \bullet \prod_{i}\left(\sum_{\begin{array}{c}
w \in W^{\prime} \\
l(w) \leq-\operatorname{deg}\left(\tau_{i}\right) \\
\operatorname{deg}(w \rho-\rho)=\operatorname{deg} \tau_{i}
\end{array}}(-1)^{l(w)+1} K_{w \rho-\rho, \tau_{i}}\right)^{t_{i}},
\end{aligned}
$$

where $\mu$ denotes the classical Möbius function.

Proof. Since

$$
\sum_{k=0}^{\infty}(-1)^{k} \operatorname{ch}\left(\wedge^{k}\left(\mathcal{L}^{-}\right)\right)=\prod_{\alpha \in \Delta_{\neq 0}^{-}}(1-e(\alpha))^{\operatorname{dim} \mathcal{L}_{\alpha}^{-}}
$$

it follows from (4.1) that

$$
\prod_{\alpha \in \Delta_{\not 0}^{-}}(1-e(\alpha))^{\operatorname{dim} \mathcal{L}_{\alpha}^{-}}=1-\operatorname{ch}(M)
$$

Thus,

$$
\prod_{\alpha \in \Delta_{\neq 0}^{-}}(1-e(\alpha))^{-\operatorname{dim} \mathcal{L}_{\alpha}^{-}}=\frac{1}{1-\operatorname{ch}(M)}=\left(1-\sum_{i}\left(\operatorname{dim} M_{\tau_{i}}\right) e\left(\tau_{i}\right)\right)^{-1}
$$

Now taking the formal logarithm of both sides and using the series expansion $\log (1-z)=-\sum_{k=1}^{\infty} \frac{1}{k} z^{k}$, we obtain

$$
-\sum_{\alpha \in \Delta_{\neq 0}^{-}}\left(\operatorname{dim} \mathcal{L}_{\alpha}^{-}\right) \log (1-e(\alpha))=\sum_{m=1}^{\infty} \frac{1}{m}\left(\sum_{i=1}^{\infty}\left(\operatorname{dim} M_{\tau_{i}}\right) e\left(\tau_{i}\right)\right)^{m} .
$$

Hence, 


$$
\begin{aligned}
\sum_{\alpha \in \Delta_{\neq 0}^{-}}\left(\operatorname{dim} \mathcal{L}_{\alpha}^{-}\right) \sum_{k=1}^{\infty} \frac{1}{k} e(k \alpha)=\sum_{m=1}^{\infty} \frac{1}{m} \sum_{\substack{t=\left(t_{i}\right) \\
m=\sum_{i} t_{i}}} \frac{\left(\left(\sum_{i} t_{i}\right)-1\right) !}{\prod_{i}\left(t_{i} !\right)} \\
\cdot \prod_{i}\left(\operatorname{dim} M_{\tau_{i}}\right)^{t_{i}} e\left(\sum_{i} t_{i} \tau_{i}\right) \\
=\sum_{\tau} \sum_{\underline{t}=\left(t_{i}\right) \in T(\tau)} \frac{\left(\left(\sum_{i} t_{i}\right)-1\right) !}{\prod_{i}\left(t_{i} !\right)} \prod_{i}\left(\operatorname{dim} M_{\tau_{i}}\right)^{t_{i}} e(\tau) \\
=\sum_{\tau} B(\tau) e(\tau) .
\end{aligned}
$$

Comparison of the coefficient of $e(\tau)$ on both sides gives

$$
B(\tau)=\sum_{\alpha \mid \tau} \frac{\alpha}{\tau} \operatorname{dim} \mathcal{L}_{\alpha}^{-}
$$

Therefore, by Möbius inversion we obtain

$$
\operatorname{dim} \mathcal{L}_{\alpha}^{-}=\sum_{\tau \mid \alpha} \mu\left(\frac{\alpha}{\tau}\right) \frac{\tau}{\alpha} B(\tau)
$$

as desired. The rest comes from substituting the expression in (4.8).

To illustrate the result in Theorem 4.12 we calculate the multiplicity of $\alpha=-\left(5 \alpha_{0}+4 \alpha_{1}+\alpha_{2}\right)=6 \epsilon_{1}+3 \epsilon_{2}+\epsilon_{3}$ using it. This multiplicity has already been computed in Example 3 of Section 3 by the methods of that section.

The Weyl group of $G=g \ell(n+1, \mathbb{C})$ is the symmetric group $W=S_{n+1}$, which acts on the weights by sending $\theta=\sum_{i=1}^{n+1} \theta_{i} \epsilon_{i}$ to $\sum_{i=1}^{n+1} \theta_{2} \epsilon_{w i}$ for $w \in W$. Thus, each weight $\theta$ is conjugate to a unique dominant weight $\bar{\theta}$ whose coefficients relative to the basis $\epsilon_{1}, \epsilon_{2}, \ldots, \epsilon_{n+1}$ are in descending order, hence form a partition. If $V(\lambda)$ is the finite dimensional irreducible $G$-module with highest weight $\lambda$, then for any weight $\theta$ of $V(\lambda), \operatorname{dim} V(\lambda)_{\theta}=K_{\lambda, \bar{\theta}}$, the Kostka number.

Example 4.13. Let $\alpha=-\left(5 \alpha_{0}+4 \alpha_{1}+\alpha_{2}\right)=6 \epsilon_{1}+3 \epsilon_{2}+\epsilon_{3}$ for $I A_{3}(2,2)$. First observe that since the coefficient of $\alpha_{2}$ is -1 , the expression in Theorem 4.12 reduces to

$$
\operatorname{dim} \mathcal{L}_{\alpha}=\operatorname{dim} \mathcal{L}_{\alpha}^{-}=B(\alpha)=\sum_{\underline{t} \in T(\alpha)} \frac{\left(\left(\sum_{i} t_{i}\right)-1\right) !}{\prod_{i}\left(t_{i} !\right)} \prod_{i}\left(\operatorname{dim} M_{\tau_{2}}\right)^{t_{2}}
$$


where $T(\alpha)=\left\{\underline{t}=\left(t_{i}\right) \mid t_{i} \in \mathbb{Z}^{\geq 0}, \quad \sum_{i} t_{i} \tau_{i}=\alpha\right\}$ and $\left\{\tau_{1}, \tau_{2}, \ldots\right\}$ is an enumeration of the weights of $M=\sum_{k=1}^{\infty}(-1)^{k+1} H_{k}\left(\mathcal{L}^{-}\right)$. Now by the same arguments as in Example 4.6 we see that the terms $H_{k}\left(\mathcal{L}^{-}\right)$for $k>3$ do not contribute to the multiplicity of $\alpha$ since their weights have degree less than $\operatorname{deg}(\alpha)=-5$. Recall from Example 4.6 that

$$
\begin{aligned}
H_{1}\left(\mathcal{L}^{-}\right) & =V\left(-\alpha_{0}\right)=V\left(2 \epsilon_{1}\right)=V(\{2\}) \\
H_{2}\left(\mathcal{L}^{-}\right) & =V\left(-3 \alpha_{0}-\alpha_{1}\right)=V(\{5,1\}) \\
H_{3}\left(\mathcal{L}^{-}\right) & =V\left(-6 \alpha_{0}-3 \alpha_{1}\right) \oplus V\left(-5 \alpha_{0}-2 \alpha_{1}-\alpha_{2}\right) \\
& =V(\{9,3\}) \oplus V\left(\left\{8,1^{2}\right\}\right) .
\end{aligned}
$$

Again from degree considerations, the only modules which can contribute to (4.14) are $V(\{2\}), V(\{5,1\})$, and $V\left(\left\{8,1^{2}\right\}\right)$. The dominant weights of these $g \ell(4)$-modules and their multiplicities are displayed below:

$$
\begin{aligned}
& V(\{2\}): \begin{array}{cc}
\{2\} & \left\{1^{2}\right\} \\
1 & 1
\end{array}
\end{aligned}
$$

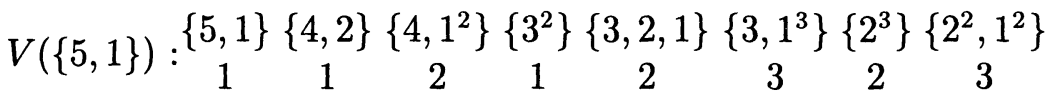

$$
\begin{aligned}
& V\left(\left\{8,1^{2}\right\}\right):\left\{8,1^{2}\right\}\{7,2,1\}\left\{7,1^{3}\right\}\{6,3,1\}\left\{6,2^{2}\right\}\left\{6,2,1^{2}\right\} \\
& \{5,4,1\}\{5,3,2\}\left\{5,3,1^{2}\right\}\left\{5,2^{2}, 1\right\}\left\{4^{2}, 2\right\}\left\{4^{2}, 1^{2}\right\} \\
& \begin{array}{llllll}
1 & 1 & 3 & 3 & 1 & 3
\end{array} \\
& \left\{4,3^{2}\right\}\{4,3,2,1\}\left\{4,2^{3}\right\}\left\{3^{3}, 1\right\}\left\{3^{2}, 2^{2}\right\} \\
& \begin{array}{llllll}
1 & 3 & 3 & 3 & 3
\end{array}
\end{aligned}
$$

These multiplicities can be computed by determining the corresponding Kostka numbers. For $\{2\}$ and $\{5,1\}$ these Kostka numbers can be found in $[\mathbf{M}]$ or ([BBL], Table (9.12)). Alternately, the multiplicities can be gotten by converting the weight to a linear combination of fundamental weights and then by consulting the appropriate table for $A_{3}$ in [BMP]. The other nondominant weights can be obtained from these by applying permutations. Note that since

$$
M=H_{1}\left(\mathcal{L}^{-}\right)-H_{2}\left(\mathcal{L}^{-}\right)+H_{3}\left(\mathcal{L}^{-}\right)-\cdots,
$$

for a weight $\tau$ of $H_{2}\left(\mathcal{L}^{-}\right)$we have $\operatorname{dim} M_{\tau}=-\operatorname{dim} V(\{5,1\})_{\tau}=-K_{\{5,1\}, \bar{\tau}}$, where $\bar{\tau}$ is the unique dominant conjugate of $\tau$. Keeping this in mind, we proceed with evaluating (4.14). Now the partitions of $\alpha$ in terms of all the weights of $M$ are: 


$$
\begin{aligned}
\alpha & =\left(6 \epsilon_{1}+3 \epsilon_{2}+\epsilon_{3}\right) \\
& =\left(5 \epsilon_{1}+\epsilon_{2}\right)+\left(\epsilon_{1}+\epsilon_{2}\right)+\left(\epsilon_{2}+\epsilon_{3}\right) \\
& =\left(5 \epsilon_{1}+\epsilon_{2}\right)+\left(2 \epsilon_{2}\right)+\left(\epsilon_{1}+\epsilon_{3}\right) \\
& =\left(5 \epsilon_{1}+\epsilon_{3}\right)+\left(\epsilon_{1}+\epsilon_{2}\right)+\left(2 \epsilon_{2}\right) \\
& =\left(4 \epsilon_{1}+2 \epsilon_{2}\right)+\left(2 \epsilon_{1}\right)+\left(\epsilon_{2}+\epsilon_{3}\right) \\
& =\left(4 \epsilon_{1}+2 \epsilon_{2}\right)+\left(\epsilon_{1}+\epsilon_{2}\right)+\left(\epsilon_{1}+\epsilon_{3}\right) \\
& =\left(4 \epsilon_{1}+\epsilon_{2}+\epsilon_{3}\right)+2\left(\epsilon_{1}+\epsilon_{2}\right) \\
& =\left(3 \epsilon_{1}+3 \epsilon_{2}\right)+\left(2 \epsilon_{1}\right)+\left(\epsilon_{1}+\epsilon_{3}\right) \\
& =\left(3 \epsilon_{1}+2 \epsilon_{2}+\epsilon_{3}\right)+\left(2 \epsilon_{1}\right)+\left(\epsilon_{1}+\epsilon_{2}\right) \\
& =\left(2 \epsilon_{1}+3 \epsilon_{2}+\epsilon_{3}\right)+2\left(2 \epsilon_{1}\right) \\
& =3\left(2 \epsilon_{1}\right)+\left(2 \epsilon_{2}\right)+\left(\epsilon_{2}+\epsilon_{3}\right) \\
& =2\left(2 \epsilon_{1}\right)+2\left(\epsilon_{1}+\epsilon_{2}\right)+\left(2 \epsilon_{2}\right)+\left(\epsilon_{1}+\epsilon_{3}\right) \\
& =\left(2 \epsilon_{1}\right)+3\left(\epsilon_{1}+\epsilon_{2}\right)+\left(\epsilon_{1}+\epsilon_{3}\right) .
\end{aligned}
$$

Hence,

$$
\begin{aligned}
\operatorname{dim} \mathcal{L}_{\alpha}^{-}= & B(\alpha) \\
= & 1+\frac{2 !}{1 ! 1 ! 1 !}(-1)(1)(1)+\frac{2 !}{1 ! 1 ! 1 !}(-1)(1)(1)+\frac{2 !}{1 ! 1 ! 1 !}(-1)(1)(1) \\
& +\frac{2 !}{1 ! 1 ! 1 !}(-1)(1)(1)+\frac{2 !}{1 ! 1 ! 1 !}(-1)(1)(1) \frac{2 !}{1 ! 1 ! 1 !}(-2)(1)(1) \\
& +\frac{2 !}{1 ! 2 !}(-2)(1)+\frac{2 !}{1 ! 1 ! 1 !}(-1)(1)(1) \frac{2 !}{1 ! 1 ! 1 !}(-2)(1)(1) \\
& +\frac{2 !}{1 ! 2 !}(-2)(1)+\frac{4 !}{3 ! 1 ! 1 !}(1)(1)(1) \frac{4 !}{2 ! 2 ! 1 !}(1)(1)(1) \\
& +\frac{4 !}{2 ! 1 ! 1 ! 1 !}(1)(1)(1)(1)+\frac{4 !}{1 ! 3 ! 1 !}(1)(1)(1) \frac{2 !}{1 ! 1 ! 1 !}(-2)(1)(1) \\
= & 1-2-2-2-2-2-4-2-2-4-2+4+6+12+4 \\
= & 3 .
\end{aligned}
$$

Concluding Remarks. The results of this paper pertain to the algebras $I A_{n}(a, b)$, but the methods for obtaining the recursive and closed form formulas work in general. The only place where the particular nature of the algebra $I A_{n}(a, b)$ is used is in evaluating the multiplicities in terms of Kostka numbers.

Although the examples presented are for the indefinite algebra $I A_{3}(2,2)$, the multiplicities of the roots computed are the same for all algebras $I A_{n}(2,2)$ 
with $n \geq 3$ and for the analogous algebras $I B_{n+1}(2,2), I C_{n+1}(2,2)$, and $I D_{n+1}(2,2)$ with $n \geq 3$ (See [BKM2], Sections 4 and 5.). These algebras are constructed by replacing the Cartan matrix $C\left(A_{n}\right)$ with the Cartan matrix corresponding to the simple Lie algebra of type $B_{n+1}, C_{n+1}$ or $D_{n+1}$.

Acknowledgment. We would like to thank Professor Stephen Doty for a helpful conversation.

\section{References}

[BBL] G.M. Benkart, D.J. Britten, and F.W. Lemire, Stability in Modules for Classical Lie Algebras - A Constructive Approach, Mem. Amer. Math. Soc., 430 (1990).

[BKM1] G. Benkart, S.-J. Kang, and K.C. Misra, Graded Lie algebras of Kac-Moody type, Adv. in Math., 97 (1993), 154-190.

[BKM2] _ Indefinite Kac-Moody algebras of classical type, Adv. in Math., 105 (1994), 76-110.

[BM] S. Berman and R.V. Moody, Lie algebra multiplicities, Proc. Amer. Math. Soc., 76 (1979), 223-228.

[BMP] M.R. Bremner, R.V. Moody, and J. Patera, Tables of Dominant Weight Multiplicities for Representations of Simple Lie Algebras, Pure and Applied Mathematics, 90, Marcel Dekker, New York and Basel (1985).

[CE] H. Cartan and S. Eilenberg, Homological Algebra, Princeton Univ. Press, Princeton, N.J. (1956).

[F] I.B. Frenkel, Representations of Kac-Moody algebras and dual resonance models, Applications of Group Theory in Physics and Mathematical Physics, Lectures in Applied Math., Amer. Math. Soc., 21 (1985), 325-353.

[FF] A.J. Feingold and I.B. Frenkel, A hyperbolic Lie algebra and the theory of Siegel modular forms of genus 2, Math. Ann., 263 (1983), 87-144.

[GK] O. Gabber and V.G. Kac, On defining relations of certain infinite-dimensional Lie algebras, Bull. Amer. Math. Soc., 5 (1981), 185-189.

[GL] H. Garland and J. Lepowsky, Lie algebra homology and the Macdonald-Kac formulas, Invent. Math., 34 (1976), 37-76.

[Kac] V.G. Kac, Infinite dimensional Lie Algebras, $3^{\text {rd }}$ ed. Cambridge U. Press, Cambridge (1990).

[Kan1] S.-J. Kang, Gradations and Structure of Kac-Moody Lie Algebras, Ph.D. dissertation, Yale University (1990).

[Kan2] Kac Moody Lie algebras, spectral sequences, and the Witt formula, Trans. Amer. Math. Soc., 339 (1993), 463-495 .

[Kan3] _ Root multiplicities of Kac-Moody algebras, Duke Math. J., 74 (1994), 635666.

[Le] J. Lepowsky, Lectures on Kac-Moody Algebras, Université Paris VI, (1978).

[Li] L.-S. Liu, Kostant's formula for Kac-Moody Lie Algebras, J. Algebra, 149 (1992), 155-178. 
[M] I.G. Macdonald, Symmetric Functions and Hall Polynomials, $2^{\text {nd }}$ ed., Clarendon Press, Oxford (1995).

[S] B.E. Sagan, The Symmetric Group, Wadsworth \& Brooks/Cole Math. Series, Belmont, CA. (1991).

Received February 25, 1993. The first author was supported in part by NSF Grant \#DMS9025111, the third author was supported in part by NSA/MSP Grants \#MDA 90-H-4039 and \#MDA 92-H-3076.

UNIVERSITY OF WISCONSIN

MADISON, WI 53706-1388

E-MAIL ADDRESS: BENKART@MATH.WISC.EDU

AND

College of Natural Sciences

Seoul National University

SEOUl 151-742, KorEa

E-MAIL ADDRESS: SJKANG@MATH.SNU.AC.KR

AND

North Carolina State University

RALEIGH, NC 27695-8205

E-MAIL ADDRESS: MISRA@MATH.NCSU.EDU 


\section{CONTENTS}

N. Ben Amar, Tangential deformations on the dual of nilpotent special Lie

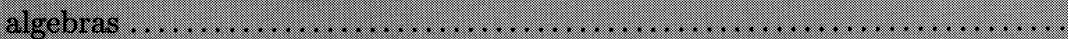

Martin Bendersky, Donald M. Davis and Mark Mahowald, $v_{1}$-periodic

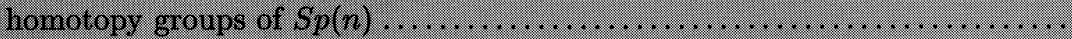

Georgia Benkart, Seok-Jin Kang, Kailash C. Misra, Indefinite Kac-

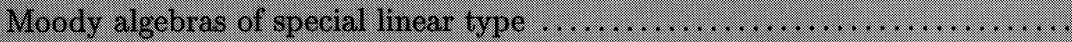

Robin Brooks and Charles Odenthal, Nielsen numbers for roots of maps

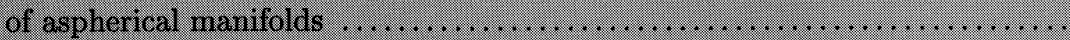

L.J. Bunce and J.D. Maitland Wright, Velocity maps in von Neumann

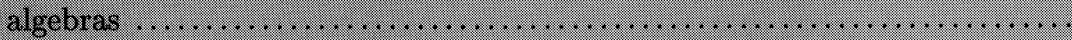

Bradley N. Currey, Smooth decomposition of finite multiplicity monomial representations for a class of completely solvable homogeneous spaces ...... R.J. Daverman and D.F. Snyder, On proper surjections with locally triv-

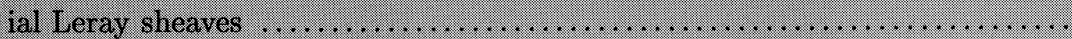

Patrick N. Dowling, Zhibao Hu and Mark A. Smith, MLUR renormings

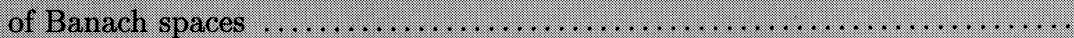
Tuval Foguel, Finite groups with a special 2-generator property ..........

Mourad E.H. Ismail and Mizan Rahman, Some basic bilateral sums and

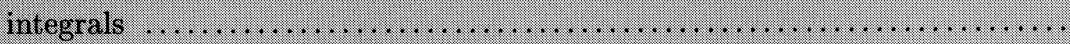

Wojciech Jaworski, Strong approximate transitivity, polynomial growth, and spread out random walks on locally compact groups $\ldots \ldots \ldots \ldots \ldots . . . . . .$.

N. Kutev and F. Tomi, Nonexistence and instability in the exterior Dirichlet problem for the minimal surface equation in the plane............

A. Nobile, Equisingularity Theory for Plane Curves With Embedded

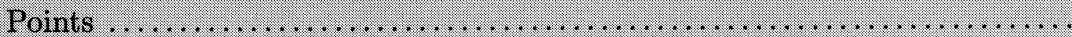

Dominikus Noll, Directional differentiability of the metric projection in Hilbert space 


\section{PACIFIC JOURNAL OF MATHEMATICS}

Volume $170 \quad$ No. $2 \quad$ October 1995

Tangential deformations on the dual of nilpotent special Lie algebras

297

NABIHA BEN AMAR

$v_{1}$-periodic homotopy groups of $S p(n)$

319

MaRTIN BENDERSKY, DONALD M. DAVIS and MARK MAHOWALD

Indefinite Kac-Moody algebras of special linear type

GeOrgia BenKart, SEOK-Jin KANG and KaILASh C. MisRa

Nielsen numbers for roots of maps of aspherical manifolds

405

ROBIN B. S. BROOKS and CHARLES ODENTHAL

Velocity maps in von Neumann algebras

L. J. BUNCE and JOHN DAVID MAITLAND WRIGHT

Smooth decomposition of finite multiplicity monomial representations for a class of 429 completely solvable homogeneous spaces

\section{BRADLEY CURREY}

On proper surjections with locally trivial Leray sheaves

ROBERT JAY DAVERMAN and DAVID FRED SNYDER

MLUR renormings of Banach spaces

PATRICK DOWLING, Zhibao Hu and MARK ANDREW SMIth

Finite groups with a special 2-generator property

TUVAL S. Foguel

Some basic bilateral sums and integrals

MOURAD ISMAIL and MIZAN RAHMAN

Strong approximate transitivity, polynomial growth, and spread out random walks on 517 locally compact groups

WOJCIECH JAWORSKI

Nonexistence and instability in the exterior Dirichlet problem for the minimal surface 535 equation in the plane

NiKolai KuteV and FriedRich TOMI

Equisingularity theory for plane curves with embedded points

Augusto Nobile

Directional differentiability of the metric projection in Hilbert space

DOMINIKUS NOLL 